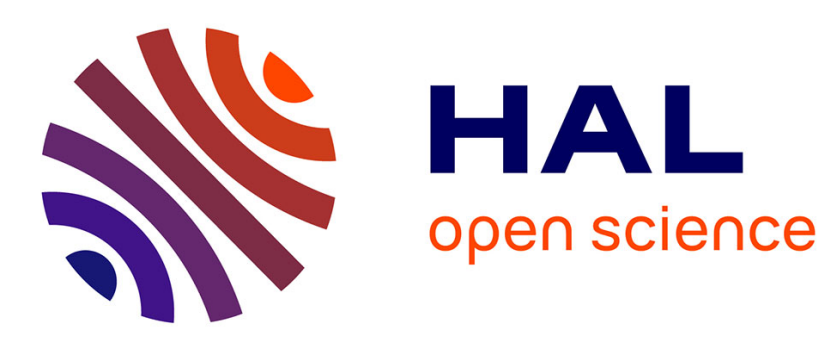

\title{
Application of X-ray diffuse scattering to structure determinations of point-defects \\ H.-G. Haubold
}

\section{To cite this version:}

H.-G. Haubold. Application of X-ray diffuse scattering to structure determinations of point-defects. Revue de Physique Appliquée, 1976, 11 (1), pp.73-81. 10.1051/rphysap:0197600110107300 . jpa00244045

\section{HAL Id: jpa-00244045 https://hal.science/jpa-00244045}

Submitted on 1 Jan 1976

HAL is a multi-disciplinary open access archive for the deposit and dissemination of scientific research documents, whether they are published or not. The documents may come from teaching and research institutions in France or abroad, or from public or private research centers.
L'archive ouverte pluridisciplinaire HAL, est destinée au dépôt et à la diffusion de documents scientifiques de niveau recherche, publiés ou non, émanant des établissements d'enseignement et de recherche français ou étrangers, des laboratoires publics ou privés. 


\title{
APPLICATION OF X-RAY DIFFUSE SCATTERING TO STRUCTURE DETERMINATIONS OF POINT-DEFECTS
}

\author{
H.-G. HAUBOLD \\ Institut für Festkörperforschung der Kernforschungsanlage Jülich 517 Jülich, Germany
}

\begin{abstract}
A review is given on the use of diffuse X-ray scattering for the determination of point defect structures in crystalline solids. Diffuse scattering yields a direct method for obtaining detailed information on the displacement fields both near to and far away from the defects in addition to information on the particular position of the defect. Theoretical models involving the use of lattice statics can be used to evaluate measurements carried out with the aid of new, high power $\mathrm{X}$-ray generators and multiple detector systems. Through the study of diffuse scattering between and close to Bragg reflections single defects such as interstitials and vacancies up to large point defect agglomerates that are visible in the electron microscope can be investigated.
\end{abstract}

1. Introduction. - In the last few years the capabilities of X-ray sources and X-ray detectors have been considerably increased. Thanks to recent technological progress, powerful X-ray sources capable of electrical loads up to $100 \mathrm{~kW}$ have been made commercially available and in addition, position sensitive detectors have been developed, which yield complete scattering patterns at one time. Through these efforts, the efficiency of X-ray methods, in measuring time, has been improved by more than a factor of $10^{4}$ [1]. This has opened new research possibilities on studies of the structure of condensed matter.

This review will be restricted to the discussions of the possibility of using diffuse X-ray scattering to solve one very old problem : The structure determination of point defects in metals. X-ray methods seem to be the most useful here because research methods such as electron spin resonance and optical methods, which have been very powerful tools for the investigation of point defects in insolators, can not be used in metallic systems.

Static defect structures can be determined by an elastic scattering experiment, which is schematically illustrated in figure 1 . The incoming $\mathrm{X}$-rays with the

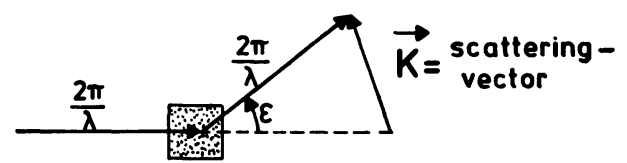

FIG. 1. - Scattering vector for elastic scattering. wavelength $\lambda$ fall on the sample and are scattered by the electrons within this sample through an angle $\varepsilon$, the wavelength remaining unchanged.

The scattered intensity depends only on the so called scattering vector $K$. This vector is the difference of the wavevectors $2 \pi / \lambda$ of the incoming and the scattered beams.

The scattered intensity from an atom decreases with increasing scattering vector as illustrated in figure 2 . It falls off similarly as X-ray scattering from a sphere which is filled uniformly with electrons.

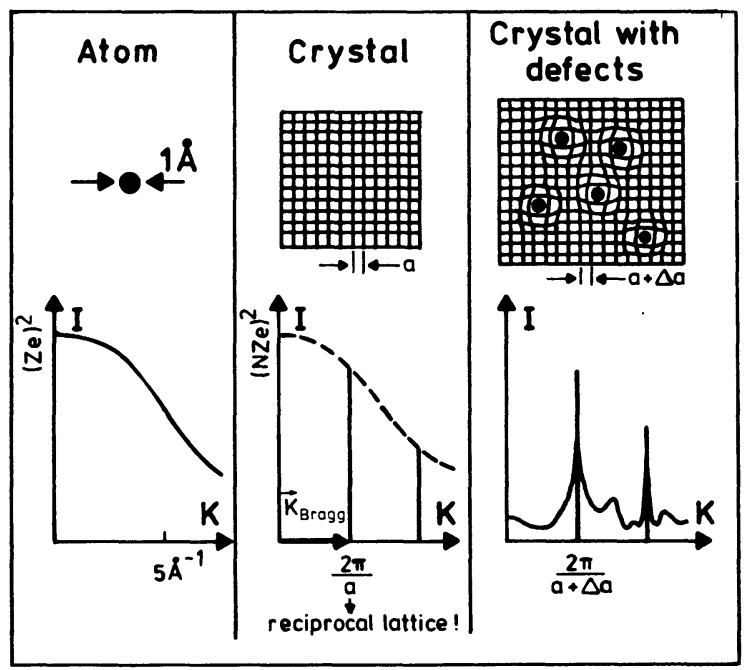

FIG. 2. - Elastic X-ray scattering. 
In a perfect crystalline solid atoms are ordered on lattice planes. As a consequence, the atomic scattering amplitudes interefere destructively at most scattering vectors. At a few scattering vectors $X$-ray are Bragg reflected from these lattice planes. These Bragg vectors also form a lattice. It is called the reciprocal lattice, since its lattice parameter $2 \pi / a$ is reciprocal in magnitude to that of the crystal noted in figure 2 as $a$.

The topic of this review, however, will not be perfect crystals but crystals with defects in them, for instance point defects as self-interstitials, vacancies, impurity atoms on lattice or interstitial sites or agglomerates of point defects.

Such defects, shown in figure 2 as black dots, will deform the lattice planes. As a consequence, the Bragg intensities decrease. This is described by a Debey Waller factor which is similar to that for thermal vibrations of the lattice atoms. Moreover the defect exerts forces on the neighbouring lattice atoms which on the average act like an internal pressure which changes the lattice parameter and shifts the Bragg reflections.

Between the Bragg reflections all scattering amplitudes cannot cancel out totally to zero intensity: A diffuse intensity remains due to the defect scattering itself and due to the displacement disorder of the lattice atoms. In figure 2 its intensity is shown strongly enlarged.

In the last few years measurement of this diffuse scattering has provided a powerful tool for the study of lattice defects.

Before some typical experimental results are given, a short introduction into the principle of the method itself will be given.

2. Principle of the method. - In figure 3 a small part of the distorted lattice is shown. By a defect on an interstitial site the lattice atoms are pushed a vector $\mathbf{S}_{\boldsymbol{n}}$ away from their lattice sites $\mathbf{r}_{n}$. The scattering amplitudes of the defect and lattice atoms are $f_{\mathbf{D}}$ and $f_{n}$ respectively.

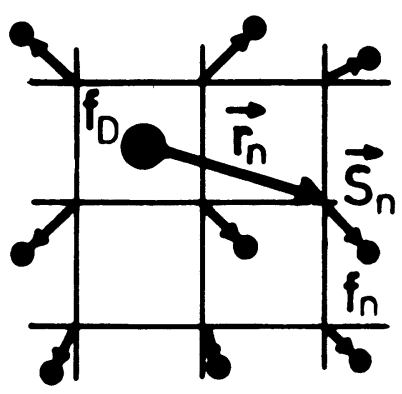

FIG. 3. - Atomic displacements around defects.

For small defect concentrations one can assume that the defects are distributed statistically. Ordering of defects would produce additional effects on diffuse scattering which will not be discussed here.

The diffuse intensity $I_{\mathrm{D}}$ is then proportional to the number $N_{\mathrm{D}}$ of defects and to the square of the diffuse scattering amplitude due to one defect.

$$
I_{\mathbf{D}}=N_{\mathbf{D}}\left|f_{\mathbf{D}}+\sum_{n} f_{n} \mathrm{e}^{i \mathbf{K}\left(\mathbf{r}_{n}+\mathbf{S}_{n}\right)}-\sum_{n} f_{n} \mathrm{e}^{i \mathbf{K} \mathbf{r}_{n}}\right|^{2}
$$

$f_{\mathrm{D}}$ is the atomic scattering amplitude of the defect itself.

$$
\sum_{n} f_{n} \exp \left[i \mathbf{K}\left(\mathbf{r}_{n}+\mathbf{S}_{n}\right)\right]
$$

is the amplitude from all lattice atoms $n$ within the distorted lattice. The phase factors give the correct phases for all scattered waves. Since the diffuse defect scattering is scattering without Bragg reflections, they are subtracted in eq. (1) as

$$
\sum_{n} f_{n} \exp \left(i \mathbf{K} \mathbf{r}_{n}\right)
$$

which is the amplitude from the lattice atoms on perfect lattice sites $\mathbf{r}_{n}$.

First some remarks will be made on the defectscattering itself. In the case of an interstitial atom, which was assumed as a defect in eq. (1), it is identical to the atomic scattering of the interstitial itself. In the case of a substitutional defect this scattering is the difference $f_{\mathrm{D}}-f$ between the scattering amplitudes from defect- and lattice-atom.

From the scattering contribution of the lattice atoms, information on the structure of the single defect displacements can be obtained. Often the product $\mathbf{k} . \mathrm{S}_{n}$ is small and an expansion yields as a first order term in the displacements :

$$
\begin{aligned}
I_{\mathbf{D}} & =N_{\mathbf{D}}\left|f_{\mathbf{D}}+f i \mathbf{K} \sum_{n} \mathbf{S}_{n} \exp \left(i \mathbf{K} \mathbf{r}_{n}\right)+\cdots\right|^{2} \\
& =N_{\mathbf{D}}\left|f_{\mathbf{D}}+f i \mathbf{K S}(\mathbf{K})+\cdots\right|^{2} .
\end{aligned}
$$

One sees, what is really measured with diffuse scattering in addition to the defect scattering itself is the projection on the scattering vector $\mathbf{K}$ on the vector $\mathbf{S}(\mathbf{K})$, which is the fouriertransform on the lattice displacements $\mathbf{S}_{n}$.

A discussion of this displacement field contribution can be simplified, since the fouriertransform $\mathbf{S}(\mathbf{K})$ is periodic in reciprocal space. This periodicity is illustrated schematically in figure 4.

To the left of figure 4, lattice displacements around a defect, which has the form of a molecule, are illustrated as arrows for various distances from the defect.

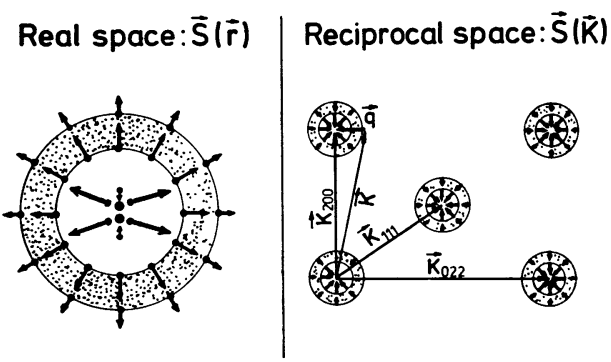

Fig. 4. - Lattice displacements in real and reciprocal space. 
In larger distances $r$ the magnitudes of the displacements fall off for point defects as :

$$
S(r \rightarrow \infty) \sim \frac{1}{r^{2}}
$$

To the right of figure 4 a picture of the fouriertransform $\mathbf{S}(\mathbf{K})$ of the displacement field is given. The displacement field is imaged around each scattering vector at which a Bragg reflection appears. In the figure these images are around the Bragg reflections in the (110)plane of a bcc reciprocal lattice and also around the origin. For an illustration of this periodicity a special region of the lattice around the defect is marked by small dots.

The displacements of the near neighbours are transformed into the region between the Bragg reflections, whereas the long ranging part of the displacement field is fourier-transformed into the close neighbourhood of the Bragg reflections. The dotted region corresponds to a distance of about $10 \AA$. The decrease of the displacements with increasing distance from the defect corresponds to an increase of their fouriertransform close to the Bragg reflections. In the following the vector $q$ is used as a measure of the distance from the scattering vector to the nearest Bragg reflection. The $1 / r^{2}$ decrease of the magnitude of the displacements in real space corresponds a $1 / q$-increase in the scattering amplitude in the neighbourhood of the Bragg reflections :

$$
S(q \rightarrow 0) \sim \frac{1}{q} .
$$

In figure 5 is illustrated, how the symmetry of the long ranging displacement field affects the intensity of the diffuse scattering near Bragg reflections.

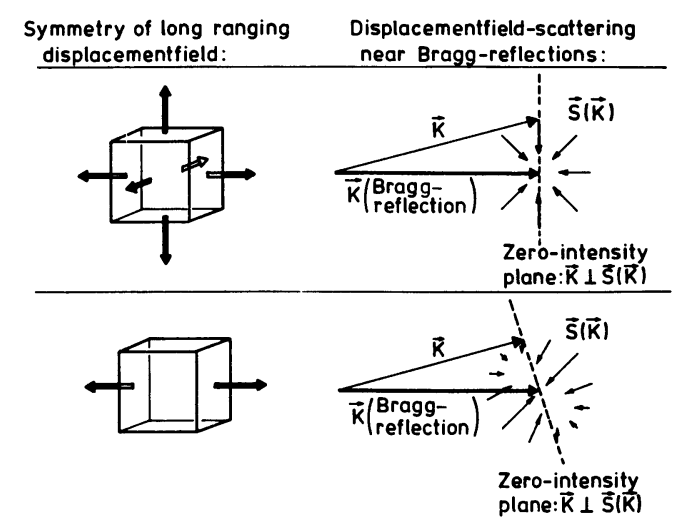

FIG. 5. - Displacement field symmetry and diffuse scattering near Bragg reflections.

A sample with isotropic defects, that means defects which produce isotropic displacement fields, is shown in the upper part. Then around each Bragg reflection the vectors of the fouriertransform of this displacement field will also be isotropic. On the dotted lined plane all vectors $\mathbf{S}(\mathbf{K})$ are perpendicular to the scattering vector
$\mathbf{K}$, thus making the product $\mathbf{K} \cdot \mathbf{S}(\mathbf{K})=0$. That means the displacement field of isotropic defects will not scatter on this zero intensity plane, that is perpendicular to the Bragg-reflection scattering vector.

Anisotropic displacement fields yield anisotropic fouriertransforms, as indicated in the lower part of the figure. This anisotropic displacement field yields therefore a zero intensity plane which is no longer perpendicular to the reciprocal lattice vectors.

Such anisotropic displacement fields will be produced from oriented anisotropic defects. Normally anisotropic defects are not oriented. Then diffuse intensity is the average of the intensity contributions from defects which have different orientations in the lattice. Each orientation can yield a different zero intensity plane. Only if they happen to fall together, is a zero intensity plane observed. Generally, two planes will intersect in 1 line, giving zero intensity and three planes will only intersect in the reciprocal lattice point, thus giving intensity in all directions around the Bragg reflection.

Trinkaus [2] and Dederichs [3] have performed such averaging processes for various kinds of anisotropy. Their results are schematically shown in figure 6 for various interstitial types in fcc lattices as examples. From symmetry arguments all interstitial sites are possible.

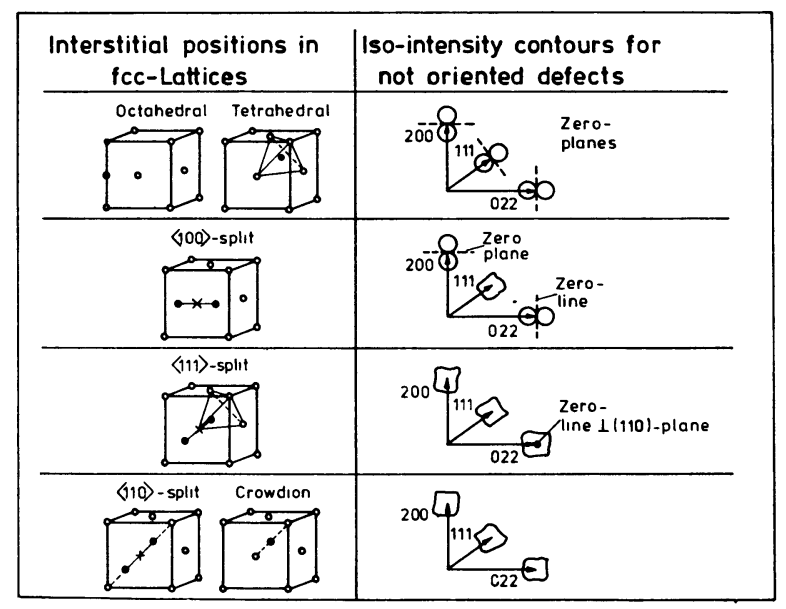

FIs. 6. - Investigation of interstitial positions from diffuse scattering near Bragg reflections.

Interstitials in octahedral and tetrahedral sites produce long ranging displacement fields with the symmetry of the cubic lattice. Zero intensity planes are therefore observed around all Bragg reflections, as shown at the 200-, 111- and 022-reflection in a (110)-plane of reciprocal lattice. Full drawn, the corresponding isointensity lines around these reflections are plotted schematically.

Anisotropic defects are the other interstitials : In the $<100>$-split interstitial the interstitial has pushed one lattice atom away from its lattice site, thus forming a dumbbell configuration with the dumbbell axis 
along [100]. The resulting displacement field slightly anisotropy is along [100]. As a result of the averaging process over the three orientations of the dumbbell axis the zero intensity plane remains only at the 200 reflection. For the other reflections the zero intensity plane vanishes or degenerates into a zero line.

The $<111>$-split, having an anisotropy along [111], yields only one zero line, whereas $<110>$-splits and crowdions, having [110]-asymmetries, give full intensity around all Bragg reflections.

Thus from a measurement of the diffuse intensity around these three reflections the symmetry and strength of the long ranging displacement field can be obtained. In addition the displacement field in the near neighbourhood is obtained from scattering between Bragg reflections. From those experiments a decision between several possible interstitial sites is therefore possible.

This is a large step forward in information on interstitials, since up till now little direct information about interstitial structures has been available. For instance in many cases the particular lattice site occupied by the interstitials is still unknown and the lattice displacements are also not very well known.

To demonstrate the method experimentally an example from radiation damage is given. The next chapter concerns with the history of structure determination of self-interstitials in aluminum, which were produced by electron irradiation.

3. Structure determination of single point defects. Results on self interstitials. - In figure 7 a measurement of diffuse scattering in the neighbourhood of the
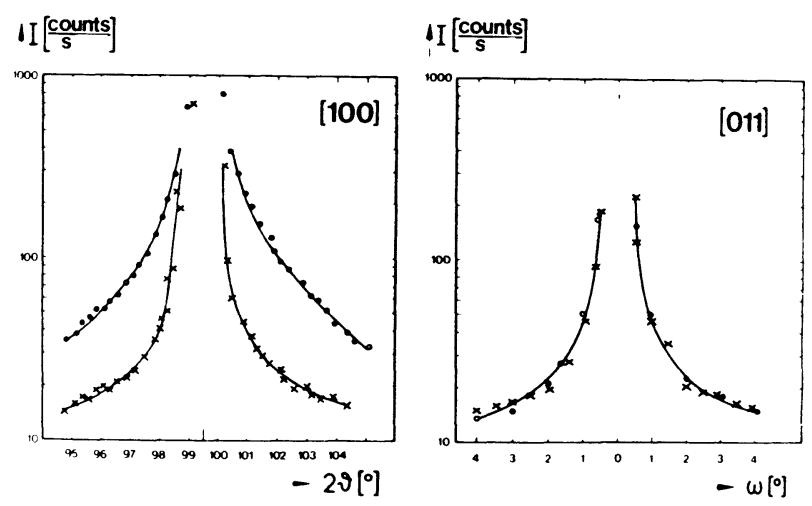

FIG. 7. - Diffuse scattering intensity measured at $5 \mathrm{~K}$ near 400 Bragg refiection in $q$-directions [100] and [011] of electron irradiated aluminum [4].

Bragg reflection is shown. It was taken from Ehrhart and Schillings paper [4] on diffuse scattering from aluminum, that contained some $10^{-4}$ atomic fractions interstitials. They measured the diffuse scattering around the 400-Bragg reflection. The measurements were carried out along the [100]-direction and perpendicular to this direction along [011]. The measured intensities along these directions are shown on a logarithmic scale for both sides of the Bragg reflection.
The crosses represent data points that were taken before the irradiation with the defect free sample and represent mainly thermal diffuse scattering due to the thermal vibrations of the lattice atoms. After the interstitials were introduced by electron-irradiation the data points represented by the circles were measured. An increased scattering was measured along the [100]-direction. Along the perpendicular direction no additional scattering was observed, indicating that a zero intensity plane exists, which is typical for defects with isotropic long ranging displacementfields.

From the possible interstitial types, shown in figure 6, the $<111>$-split, $<110>$-split and crowdion configurations can thus be ruled out. A decision between the isotropic octahedral and tetrahedral sites and the anisotropic $<100>$-split interstitial configuration could not be derived from diffuse scattering near Bragg reflections alone, since the displacement field anisotropy of the $<100>$-split interstitial is very small at large distances from the defect.

For a further determination one has to look for differences in the displacements in the neighbouring lattice atoms, which can be measured between the Bragg reflections. Even though the long range displacement fields are similar, the near displacements are strongly different for these remaining configurations as indicated by the forces, $\mathbf{f}$, shown in figure 8 for the nearest neighbour atoms.

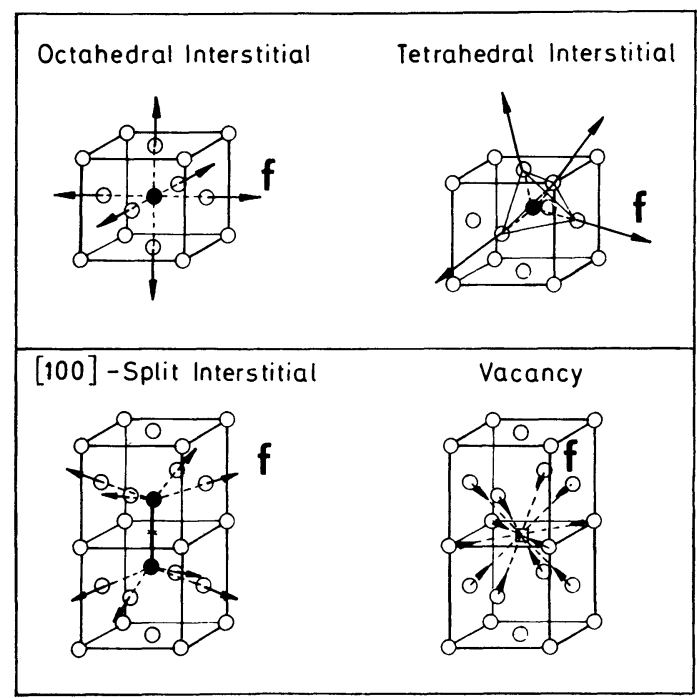

FIG. 8. - Simplest force models for point defects.

A final determination of the interstitial type should therefore be possible by comparing measured intensities in the region between the Bragg reflections with calculated intensities for interstitials with these three configurations.

The diffuse scattering can be calculated easily assuming the simple force models with forces acting only on nearest neighbours. This is a good approximation, since in spite of the long range of the displacements the forces are highly restricted to the neighbouring atoms 
only, because in metals the electrons screen out the defect potential very efficiently at larger distances.

The magnitude of the forces $\mathbf{f}_{n}$, acting on neighbours with distance $\mathbf{r}_{n}$ from the defect, can be evaluated from the known lattice parameter change per defect $\Delta a / a$ according to eq. (5), which is valid for unoriented defects in cubic lattices [3] :

$$
\sum_{n} \mathbf{r}_{n} \mathbf{f}_{n}=3 \frac{\Delta a}{a}\left(c_{11}+2 c_{12}\right) V_{\mathrm{A}}
$$

$c_{11}$ and $c_{12}$ are elastic constants, $V_{\mathrm{A}}$ is the atomic volume of one lattice atom. From these forces the atomic displacements are obtained through lattice statics :

$$
S_{n, i}=\sum_{m, j} G_{n m, i j} f_{m, j}
$$

where $G_{n m, i j}$ is the response function of the lattice and gives the displacement $S_{n, i}$ of atom $n$ in the $i$-direction, for a unit force acting on atom $m$ in the $j$-direction. The response function can be calculated from dispersion curves, which have been measured very precisely in most materials with inelastic neutron scattering.

For a calculation of the diffuse scattering the fouriertransform $\mathbf{S}(\mathbf{K})$ is needed. This is given by a product of the fouriertransforms of forces and responsefunction :

$$
S_{i}(\mathbf{K})=\sum_{j} G_{i j}(\mathbf{K}) f_{j}(\mathbf{K}) .
$$

Results of a numerical calculation for these interstitials are shown in figure 9. Diffuse scattering intensities in the (110)-plane of the reciprocal lattice are given in

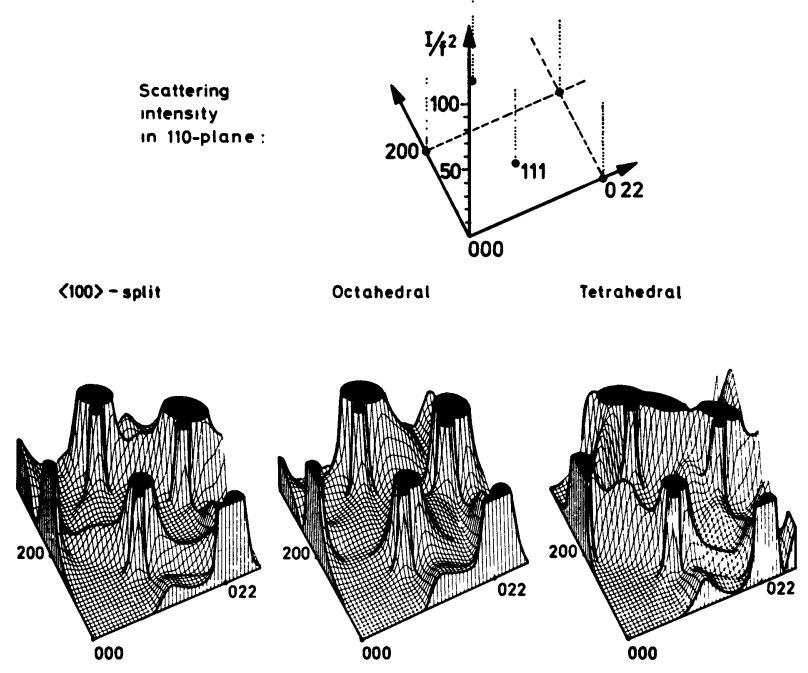

FIG. 9. - Calculated diffuse scattering intensities from interstitials in aluminum [5].

three dimensional plots. In the upper part of the figure the positions of the Bragg peaks are indicated. In the lower part, the diffuse scattering intensities for the $<100\rangle$-split, the octahedral and the tetrahedral configurations are shown for values, which go up to about 60 times higher than that for the scattering of the interstitials themselves. This high intensity is caused by the additional scattering of the displaced lattice atoms surrounding the defect.

Because of their nearly identical long ranging displacement fields the diffuse scattering very close to the Bragg reflections, not shown in these plots, do not differ for these three configurations. Almost equal intensity distributions are observed in figure 9 also at slighty larger distances from the reflections, which are most sensitive to displacement order of about $20 \AA$ from the defects.

However, because of the different near neighbour displacements, the diffuse intensity differs strongly in the region between the Bragg reflections, especially between the 200- and 111-reflection. These differences are mainly due to the position of the defect within the lattice and are not very sensitive to the details of the force model that was used for the calculation. All three interstitial configurations can therefore be determined quite unambigously from this diffuse scattering between the Bragg reflections.

Unfortunately in this region the backgroundscattering, mostly Compton-scattering, is up to 100 times larger than the scattering of typically $500 \mathrm{ppm}$ interstitials.

It is in most cases impossible to enhance the defect scattering by higher defect concentrations, because at higher concentrations agglomeration processes can mask the effects of the single defects.

These severe background problems are then the reason why such X-ray measurements of the scattering from single interstitials in the region between the Bragg reflections have not been done before : Usual X-ray set ups yield too long measuring times to make statistical errors small enough for a subtraction of the background scattering which can be measured with a defect free sample. Those measurements become possible however, if special designs are used.

In figure 10 an arrangement is shown, which was optimized for measurements of defect diffuse scattering between the Bragg reflections. Compared with conventional set-ups, about a factor of $10^{4}$ in the measuring time is gained. This factor results first from using a $100 \mathrm{~kW}$ rotating anode X-ray tube instead of a $1 \mathrm{~kW}$

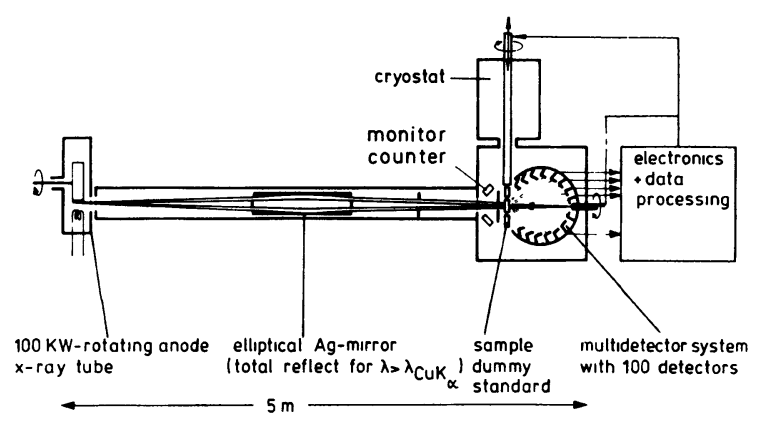

Fig. 10. - Measuring system for the diffuse scattering between reciprocal lattice points [5]. 
tube, and second from using a multicounter system with 100 detectors instead of only 1 detector. These detectors, which are arranged on a focusing circle, feed the incoming data online into a data processing system.

The samples are typically single crystals of $1 \mathrm{~cm}^{2}$ with thicknesses of some $10-100 \mu \mathrm{m}$. The measurements are normally performed at $4 \mathrm{~K}$ to prevent the defects from becoming mobile and agglomerating into clusters. To make highly accurate cross section measurements possible, a differential technique is applied. Each $3 \mathrm{~min}$. the sample, containing the defects, is interchanged with a dummy sample. In this manner within typical measuring times of several hours fluctuations in counting efficiencies and primary beam intensity have no influence on the accuracy of the measurement.

In figure 11 it is illustrated that defect scattering cross sections can be measured with such special set

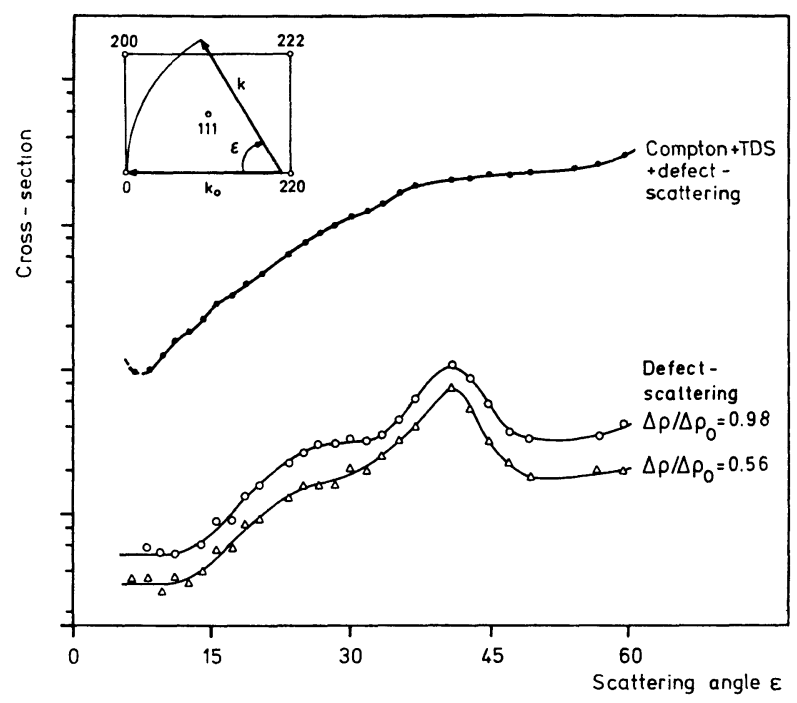

FIG. 11. - Background and defect scattering at $5 \mathrm{~K}$ from electron-irradiated aluminium (interstitial concentration $340 \mathrm{ppm})$ [5]. ups, even if the background scattering is very high. In a logarithmic scale both the background and defect scattering are shown as a function of the scattering angle for aluminum with about $300 \mathrm{ppm}$ interstitials, which were produced by an irradiation with $3 \mathrm{MeV}$ electrons at $4 \mathrm{~K}$. The corresponding scattering vectors lie on a circle in the (110)-plane of reciprocal space, as indicated in the insert.

The total measured intensity (mostly Compton background scattering) increases monotonically with increasing scattering angles.

Two curves are shown for defect scattering. They were derived as the difference between the scattering from the irradiated sample and the scattering from the same sample before the defects were introduced. The upper curve gives the scattering directly after irradiation. The lower curve gives the results after about half of the defects had been thermally annealed. The intensity ratio corresponds very well to the two defect concentrations. As a measure of the concentration the change of the electrical residual resistivity $\Delta \rho$ was used.

With increasing scattering angles the defect scattering increases more than a factor 10 as a consequence of the increasing influence of the displacement field scattering. In spite of this, at the largest scattering angles the background scattering is about 100 times stronger than the defect scattering itself. The defect scattering however could be measured also in this unfavourable region with accuracies better than 10 percent.

In figure 12 the defect scattering from interstitials (again in a concentration of some $10^{-4}$ atomic fractions) is shown in an extended area of reciprocal space. As indicated in the insert the measurements were done along four Ewald circles in the (110)-plane of reciprocal lattice, corresponding to measurements in which the sample has been rotated around the [110]-axis perpendicular to the incident beam.

To the left of figure 12 the experimental results are
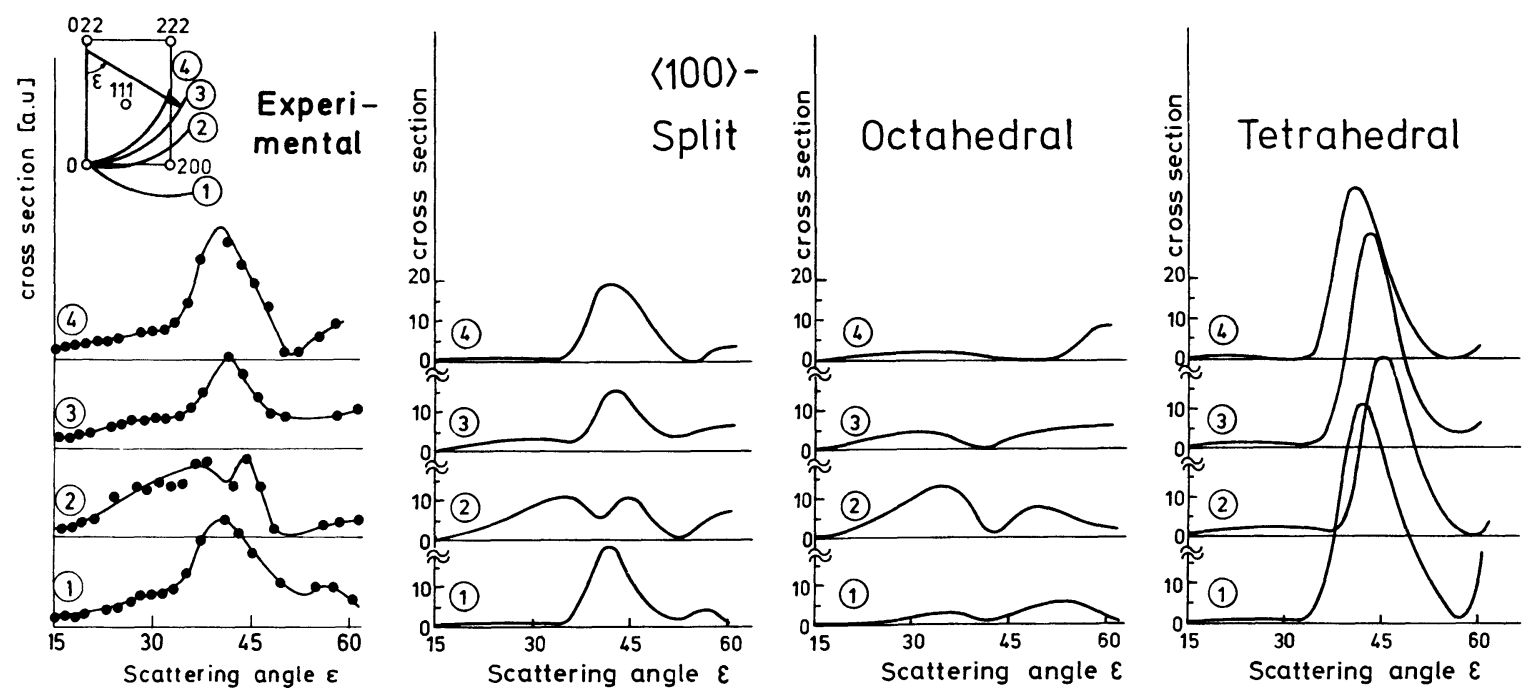

FIG. 12. - Comparison of experimental and calculated cross-sections of interstitials in electron-irradiated aluminum [5]. 
plotted, as measured along these four circles. To the right, calculated cross sections are plotted along the same circles. They are identical to cuts through the intensity distributions in figure 9. From a qualitative comparison of the scattering profiles for the $<100>$-split, the octahedral and the tetrahedral interstitial configuration with the experimental results one arrives at the conclusion that only the pattern calculated for the $\langle 100\rangle$-split interstitial agrees with the experimental data. From this the intrinsic interstitial in aluminum was clearly proven to be the $<100>$-split interstitial.

Figure 13 shows a quantitative fit of the calculated curves to the experimental data. The full lines give the

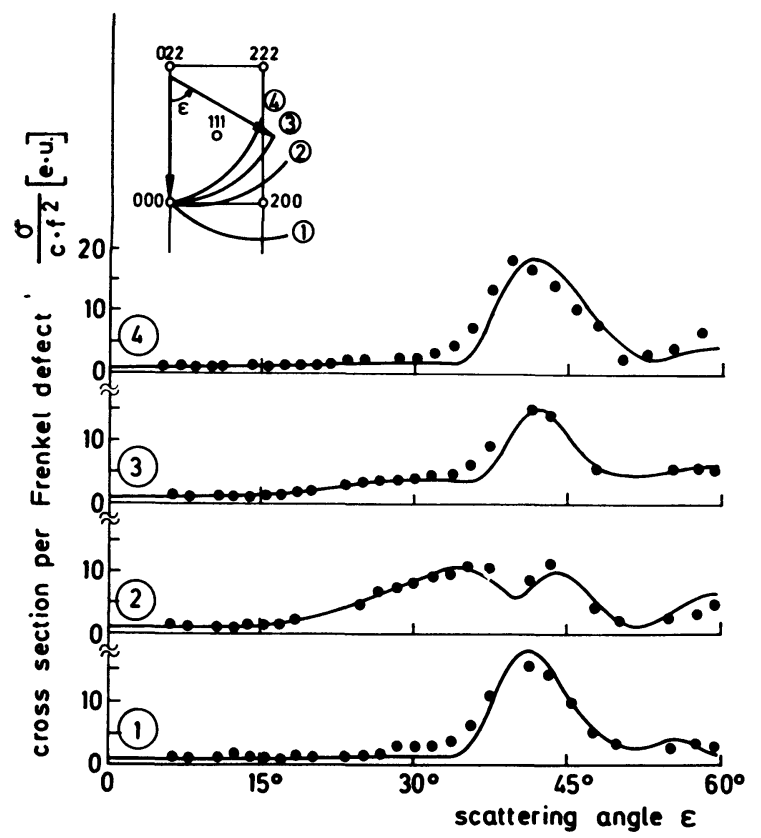

Fig. 13. - Fit of a model calculation to the diffuse-scattering intensity from $<100>$-split interstitials and vacancies to the experimental points [5].

theoretical result using optimum values for the different parameters entering the calculation. In this case the contribution of the vacancy was also included, which was produced along with the interstitial in equal number during the irradiation. Because of the smaller displacements around a vacancy, its scattering is only important at small scattering angles.

The number of parameters of the interstitial was restricted to only two parameters : dumbbell distance and central forces to only nearest neighbours. In spite of this restriction a very satisfactory fit to the experimental data was obtained.

As a result of this fit the distance between the two dumbbell atoms was found to be $0.6 \mathrm{a}$, the displacements of the nearest neighbours to be $0.1 \mathrm{a}$, and the volume relaxation around the interstitial to be 1.9 at. vol. From the scattering intensity at small scattering angles also experimental information about vacancies could be derived, for instance its volume dilatation turned out to be nearly zero.

Scattering in the small angle region is also very sensitive to small vacancy agglomerates, which produce an increase of the small angle scattering intensity. This was observed very recently in irradiated copper after annealing at a temperature of $220 \mathrm{~K}$.

4. Results on impurity interstitials. - Impurity interstitials, such as oxygen in niobium yield equally unresolved questions on the interstitial site and the lattice displacements. Diffuse scattering from this system [7] is shown in figure 14.
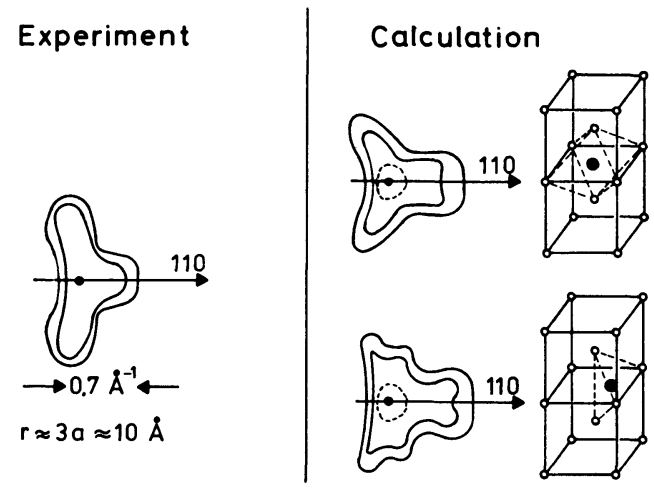

FIG. 14. - Isointensity diffuse scattering curves for 0 interstitials in $\mathrm{Nb}$ in (100) -plane around 110 reflection [7].

Iso-intensity curves are plotted in the (100)-plane of reciprocal lattice around the 110 Bragg-reflection. To the left the measurements are shown and to the right results from calculations are plotted. These were performed for oxygen in the possible octahedral- and tetrahedral positions of the bcc niobium-lattice, using force models for the impurity interstitial similar to those used in figure 8 for self interstitials in aluminum.

At large distances, oxygen produces displacement fields with tetragonal symmetry in both of octahedraland tetrahedral positions. This tetragonality was observed by diffuse scattering close to the Bragg reflections [6] and the parameters measured lead to the dotted iso-intensity lines very close to the Braggreflection.

Farther from the Bragg reflections, the scattering from atoms nearer to the oxygen becomes dominant. Because the near neighbours are displaced differently in the two models, the solid iso-intensity lines are somewhat different there. The octahedral position agrees better with the experimental measurements and this is a strong indication, that oxygen interstitials in niobium are in the octahedral configuration.

Additional measurements are in progress farther away from the Bragg reflection where larger differences in the intensity patterns for the octahedral and tetrahedral sites are to be found. The measurement given here was most sensitive to the still rather similarly displaced atoms the order of $10 \AA$ from the oxygen. 
5. Structure determination of defect agglomerates. Figure 15 gives an illustration of what happens when single point defects agglomerate into clusters. In a first
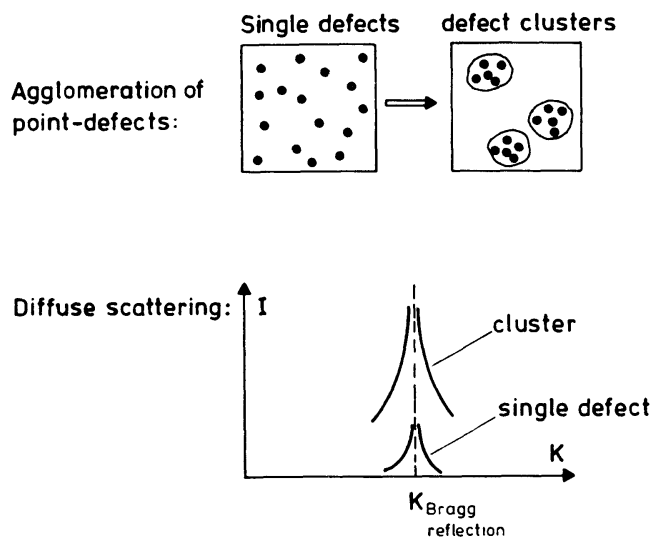

FIG. 15. - Investigation of defect clusters from diffuse scattering near Bragg-reflections.

approximation, the displacement fields of $N$ point defects in the clusters add coherently.

$$
S_{\text {cluster }}(r \rightarrow \infty) \approx N \cdot S_{\text {single defect }}(r \rightarrow \infty) .
$$

Thus at large distances the clusters appear as new strongly distorting point defects. Since the number of clusters decreases linearly with the number of defects, $N$, in a cluster while the scattering intensity of one cluster increases as the square of the displacements (i. e. with $N^{2}$ ), a very large increase in the scattering near Bragg reflections results.

For this reason it is possible to perform measurements on clustered defects with conventional X-ray devices. In figure 16 diffuse scattering from clusters in the form of precipitates and dislocation loops is shown. These measurements [8] were made near the 200 reflection of copper with cobalt precipitates and copper with dislocation loops produced by neutron irradiation.

The measured iso-intensity lines for the spherical Co-precipitates differ strongly from that for the dislocation loops, consisting of interstitials, which had been clustered to form additional lattice planes.

The spherical precipitates yield a zero intensity plane as one expects for isotropic displacement fields. As is typical for anisotropic defects, a zero plane is not observed for the dislocation loops. The reversed asymmetry in the scattering relative to the Bragg points is a direct result of the fact that the displacements are inward for cobalt precipitates and for interstitial loops are outward.

As was proved by these measurements, in addition to single point defects also defect clusters up to sizes of $150 \AA$ and larger can be studied with diffuse X-ray scattering. Thus an overlap is possible with electron microscope work, which is possible for larger defects.
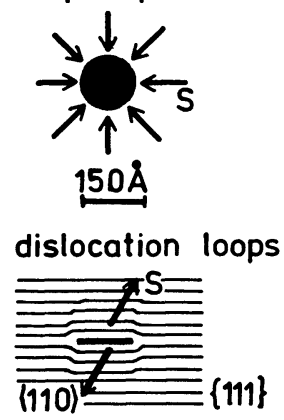

(a)
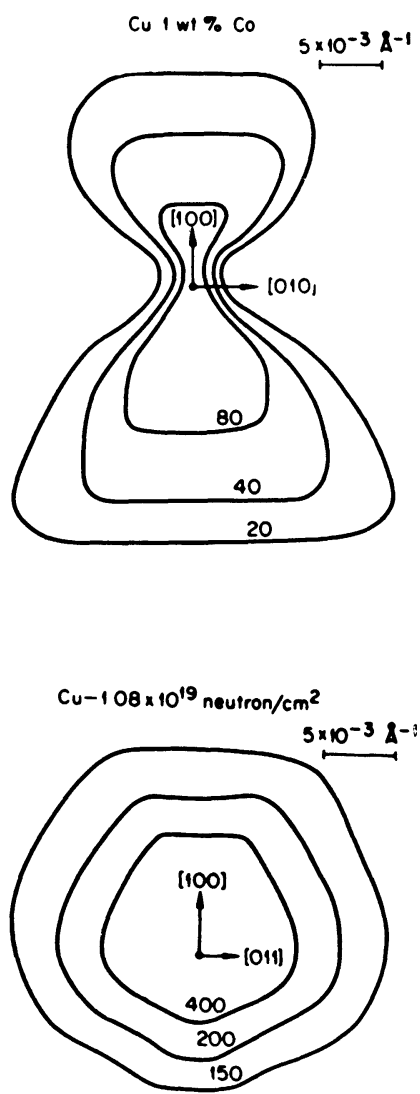

(b)

Fig. 16. - Diffuse scattering contours measured near the 200 Bragg-reflection with a three axis diffractometer [8].

6. Conclusions. - While X-ray scattering is not new, the application of X-ray diffuse scattering for detailed studies of point defects and their clusters has only recently become possible as a result of recent theoretical and experimental advances.

As was outlined, these measurements require some effort. However these efforts are justified, because X-ray diffuse scattering:

1) Allows the investigation of single defects in low enough concentration to avoid interactions ; 
2) Yields direct information on the defect structure, which is not available from other methods;

3) Can be universally applied to various kinds of defects.

To date the interest has been mainly concerned with the defect structures of strongly distorting defects.
A future application is the investigation of defects with small or no displacement fields, thus making the atomic scattering itself an object of research.

Acknowledgements. - The author would like to thank Dr B. C. Larson and Prof. Dr W. Schilling for helpful discussions and Dr B. C. Larson for carefully reading the manuscript.

\section{References}

[1] Haubold, H.-G., J. Appl. Cryst. 8 (1975) 175-183.

[2] Trinkaus, H., Phys. Stat. Solids (b) 51 (1972) 307-319.

[3] Dederichs, P. H., J. Phys. F : Metal Phys. 3 (1973) 471-496.

[4] Ehrhart, P., Schilling, W., Phys. Rev. B 8 (1974) 26042621.

[5] Haubold, H.-G. Rep. Kernforschungsanlage Jülich Jül1090-FF.
Haubold, H.-G., Schilling, W., (1976) to be published. [6] Wombacher, P., Rep. Kernforschungsanlage Jülich Jül-942FF (1972).

[7] Haubold, H.-G., Larson, B. C., Martinsen, D., to be published.

[8] Larson, B. C., J. Appl. Cryst. 8 (1975) 150-160. 